\title{
Chromosomes and cancer cells
}

\author{
Sarah L. Thompson • Duane A. Compton
}

Published online: 29 December 2010

(C) Springer Science+Business Media B.V. 2010

\begin{abstract}
Two prominent features of cancer cells are abnormal numbers of chromosomes (aneuploidy) and large-scale structural rearrangements of chromosomes. These chromosome aberrations are caused by genomic instabilities inherent to most cancers. Aneuploidy arises through chromosomal instability (CIN) by the persistent loss and gain of whole chromosomes. Chromosomal rearrangements occur through chromosome structure instability (CSI) as a consequence of improper repair of DNA damage. The mechanisms that cause CIN and CSI differ, but the phenotypic consequences of aneuploidy and chromosomal rearrangements may overlap considerably. Both CIN and CSI are associated with advanced stage tumors with increased invasiveness and resistance to chemotherapy, indicating that targeted inhibition of these instabilities might slow tumor growth. Here, we review recent efforts that define the mechanisms and consequences of CIN and CSI.
\end{abstract}

Keywords chromosome $\cdot$ aneuploid $\cdot$ CIN $\cdot$ CSI $~ \cdot$ kinetochore $\cdot$ microtubule $\cdot$ merotely $\cdot$ translocation

Responsible Editor: James Wakefield and Herbert Macgregor

S. L. Thompson · D. A. Compton $(\bowtie)$

Department of Biochemistry, Dartmouth Medical School, 405 Remsen Building,

Hanover, NH 03755, USA

e-mail: duane.a.compton@dartmouth.edu

S. L. Thompson · D. A. Compton

Norris Cotton Cancer Center,

Lebanon, NH 03766, USA

\begin{abstract}
Abbreviations
CIN Chromosomal instability

CSI Chromosome structure instability

kMT Kinetochore-microtubule
\end{abstract}

\section{Introduction}

One of the most striking features of cancer cells is the presence of chromosomal abnormalities. Aneuploidy, an abnormal number of chromosomes, is found in most solid tumors and half of all leukemias and lymphomas (http://cgap.nci.nih.gov/Chromosomes/ Mitelman). Aneuploidy is caused by an underlying erosion of mitotic fidelity called chromosomal instability (CIN) which is defined as a persistently high rate of loss and gain of whole chromosomes (Lengauer et al. 1997). Aneuploidy and CIN are associated with poor patient prognosis, metastasis, and resistance to chemotherapeutics (Kuukasjärvi et al. 1997; Gao et al. 2007; Choi et al. 2009; McClelland et al. 2009; Swanton et al. 2009; Heilig et al. 2010). In addition to changes in chromosome numbers, structural defects in chromosomes are present in a high percentage of both blood cancers and solid tumors. In leukemic and lymphomic cells, often only a single, balanced translocation is present which drives tumorigenicity (Mitelman et al. 2007). Solid tumors often contain many structural rearrangements involving multiple chromosomes, multiple breakpoints, and frequently cells from the same tumor contain 
different rearranged chromosomes, indicative of ongoing chromosome structure instability (CSI). The contribution of specific chromosomal translocations in blood cancers to tumorigenesis is well established (Mitelman et al. 2007; Nambiar et al. 2008), and CSI is associated with a large number of inherited syndromes with increased cancer risk (Duker 2002) and has been shown to cause tumors in mice (Artandi et al. 2000; Luo et al. 2000).

CIN and CSI are evident in the karyotypes of cancer cells. The chromosomal content of the U251 glioblastoma cell line using spectral karyotyping (SKY) is depicted in Fig. 1. Panels a and b are karyotypes of two cells from this cell line providing evidence for the ongoing chromosome missegregation associated with $\mathrm{CIN}$ by the numerical changes in various chromosomes between the two cells (e.g., chromosome 7 is present in five copies in panel a and only two copies in panel b). The ongoing structural changes of chromosomes associated with CSI are also shown by the structural rearrangements of various chromosomes between the two cells [e.g., rearranged chromosomes containing parts of chromosomes 11, 10, and 15 are present in the karyotype of the cell in panel a, but this is absent in the karyotype of the cell in panel $b$, while the karyotype of the cell in panel $b$ contains $t(11 ; 15)$ that is absent in the karyotype of the cell in panel a]. The genome instability depicted in this cell line is typical of many cancer cells and underscores the magnitude of ongoing genetic change that impacts cancer cells. Here, we discuss how CIN generates aneuploidy, how CSI generates structural defects, and the consequences of these chromosome aberrations on cancer cells.

\section{Mechanisms of chromosomal instability}

Whole chromosome instability occurs through missegregation of chromosomes during mitosis. Cancer cells with CIN mis-segregate a chromosome about once every one to five divisions, compared to rates of one chromosome per a hundred cell divisions in stable, diploid cell lines (Cimini et al. 1999; Thompson and Compton 2008). CIN can be mimicked in diploid cells grown in culture, elevating levels of aneuploidy through perturbations of proteins that play key roles in mitosis including proteins that maintain the mitotic spindle checkpoint and sister chromatid cohesion (Jallepalli et al. 2001; Michel et al. 2001). However, direct analysis of chromosome segregation in CIN cells shows that defects in checkpoint function are unlikely to cause CIN in human cancers. Cancer cells with CIN only enter anaphase after alignment of all chromosomes, and cells with CIN remain arrested in mitosis when exposed to microtubule perturbing drugs (reviewed in Thompson et al. 2010). Instead, evidence from human cancer cell lines suggests a specific kinetochore-microtubule (kMT) attachment error called merotely is the major source of chromosome segregation errors causing CIN (Thompson and Compton 2008). Merotelic attachments are defined by single kinetochores attaching to microtubules emanating from more than one spindle pole (Fig. 2). Merotely is not detected by the spindle checkpoint, and chromosomes with merotelic attachments align at the metaphase plate (Khodjakov et al. 1997; Cimini et al. 2002). Merotely occurs stochastically, and in early mitosis numerous chromosomes have merotelic kinetochores. These errors are corrected in normal cells prior to anaphase onset to prevent chromosome missegregation and preserve the diploid chromosome content (Cimini et al. 2002; Salmon et al. 2005). If a cell enters anaphase with a merotelic attachment, the chromatid attached to both poles can segregate to the same daughter cell as its sister resulting in a missegregation that produces two aneuploid cells, one with an extra copy of the chromosome and the other missing a copy (Fig. 2).

In human cancer cells, the persistence of merotely at the time of anaphase onset is caused by two different mechanisms, a decreased rate of error correction and an increased rate of merotelic formation. Merotelic errors are corrected by the detachment of improperly oriented microtubules from kinetochores. This correction process relies upon the reversible attachment of microtubules to kinetochores and is reflected by the overall turnover (attachment/ detachment dynamics) of kMTs. Human kinetochores bind approximately 25 microtubules, and as cells progress through mitotic stages from prometaphase to metaphase to anaphase the turnover of kMTs decreases indicative of a gradual stabilization of kMT attachments (Zhai et al. 1995; Cimini et al. 2006; Bakhoum et al. 2009a). In cancer cells with CIN, the turnover rate of kMTs is slower than in chromosomally stable, diploid cells, demonstrating that cancer cells with CIN have an inherently reduced 

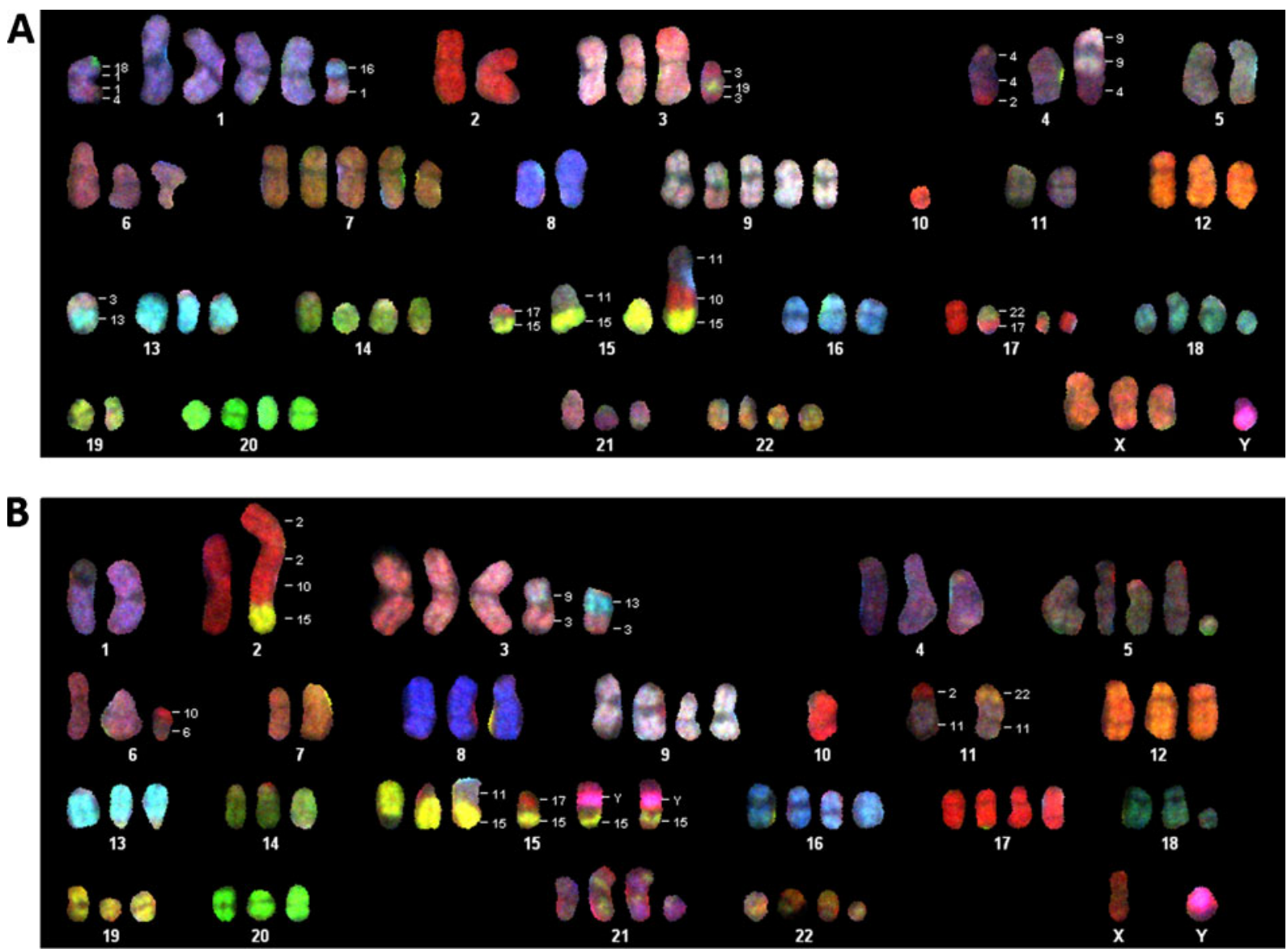

Fig. 1 Karyotype of human U251 glioblastoma cells. SKY chromosome painting of two different cells (a, b) from the same culture of U251 cells illustrating the ongoing CIN (chromosomal instability) and CSI (chromosome structure instability)

capacity to correct erroneous kMT attachments (Bakhoum et al. 2009a, b). Increasing kMT turnover by overexpression of microtubule depolymerases $\mathrm{Kif} 2 \mathrm{~b}$ and MCAK restores $\mathrm{kMT}$ turnover rates to levels found in stable diploid cells and re-establishes faithful chromosome segregation to cancer cells with
CIN (Bakhoum et al. 2009a). Depletion and overexpression of numerous kinetochore proteins that regulate microtubule attachment or stability at the kinetochore leads to an increase in merotely, including MCAK, Kif2b, Aurora B, adenomatous polyposis coli (APC), CENP-E, CLASPs, the NDC80 complex,

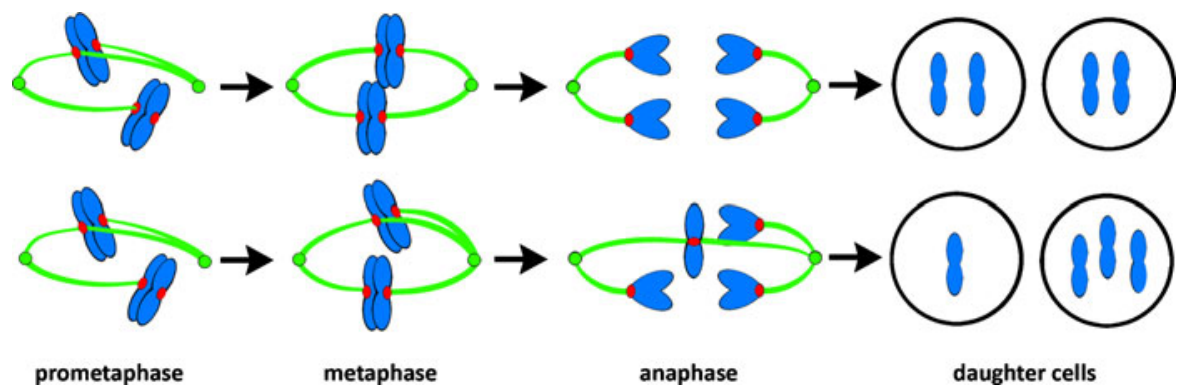

Fig. 2 Merotelic attachments lead to segregation defects. Merotely occurs stochastically in early mitosis in normal cells, but is corrected efficiently to establish proper, bi-oriented attachments needed for error-free segregation (top). When

merotelic kinetochore-microtubule attachments are not efficiently corrected during early mitosis, the chromosome attached to both spindle poles may mis-segregate during anaphase, leading to aneuploidy in both daughter cells (bottom) 
Mps1, and Mad2 (Maney et al. 1998; Yao et al. 2000; Hauf et al. 2003; Kline-Smith et al. 2004; Cimini et al. 2006; Knowlton et al. 2006; Pereira et al. 2006; Sotillo et al. 2007; Diaz-Rodriguez et al. 2008; Jelluma et al. 2008; Bakhoum et al. 2009a, b; Maffini et al. 2009). Whereas some of these proteins are mutated in some cancers (i.e., APC), acquired mutation in the others is rare as shown by genomic sequencing of hundreds of tumors (Wood et al. 2007; Jones et al. 2008; Parsons et al. 2008).

An intriguing hypothesis is that imbalances of mitotic proteins that are encoded by genes on aneusomic chromosomes actively promote CIN (Duesberg et al. 1998, 1999). For example, when otherwise diploid, chromosomally stable cells are forced to mis-segregate chromosomes for many generations, the aneuploid progeny develop high chromosome mis-segregation rates (Thompson and Compton 2010). These findings indicate that aneuploidy can cause CIN and that CIN may be a selfpropagating type of genome instability. Any imbalance in protein levels that results in suppressed kMT dynamics can, in principle, cause CIN by undermining the correction of kMT attachment errors. Given the multitude of proteins that contribute to appropriate kMT dynamics, there may be countless chromosome combinations that would promote CIN. Many of these will have direct effects on kMT stability, but some may promote CIN indirectly. For example, perturbation of regulatory proteins (e.g., FoxM1, Rb, and REST) appears to promote CIN by creating dosage imbalances in their downstream targets (Hernando et al. 2004; Laoukili et al. 2005; Guardavaccaro et al. 2008). Loss of Rb disrupts cell cycle control at the G1/S transition, but also elevates the level of merotely and reduces chromosome segregation fidelity by the alteration in the appropriate regulation of its downstream target gene expression (Hernando et al. 2004; Ferretti et al. 2010; Manning et al. 2010).

The CIN phenotype can also be caused by an increased rate of formation of merotelic kMT attachments. Any treatments that disrupt the normal geometric assembly of the mitotic spindle increase merotelic kMT formation. This can be induced experimentally by the treatment of mitotic cells with compounds that reversibly disrupt spindle formation without disrupting the endogenous correction machinery (Cimini et al. 2002, 2003; Thompson and Compton 2008). In cancer cells, the geometric assembly of spindles is disrupted by extra centrosomes which have long been linked to CIN. Centrosomes dictate the number of spindle poles formed in mitosis and there is a correlation between extra centrosomes and multipolar spindles in cancer cells (Ghadimi et al. 2000; Lingle et al. 2002). However, chromosome segregation during anaphase in cells with multipolar spindles is lethal (Kwon et al. 2008; Ganem et al. 2009). Thus, it is essential that cells evade a multipolar anaphase by clustering centrosomes to form two spindle poles (Quintyne et al. 2005). However, the multipolar spindles that form transiently in cells with extra centrosomes undermine the geometric constraints of normal kMT attachments and greatly increase the rate of merotely and subsequent chromosome mis-segregation (Ganem et al. 2009; Silkworth et al. 2009). Experiments where extra centrosomes were induced in chromosomally stable, diploid cells, which have normal levels of microtubule turnover at the kinetochore, led to increased rates of merotely and chromosome mis-segregation, suggesting that the levels of merotely generated through transient multipolar spindles overwhelms the error correction machinery (Ganem et al. 2009). Extra centrosomes arise through multiple pathways. Tetraploid or polyploid cells that arise from cytokinesis failure, fusion of two diploid cells, or endoreduplication often contain supernumerary centrosomes (Ganem et al. 2007). Centrosome amplification also occurs when the strict cell cycle-dependent regulation of their duplication is lost through perturbation of cyclin E, SAS-4, SAS-6, or Plk4 expression (reviewed in Bettencourt-Dias and Glover 2007).

Another geometric constraint that discourages merotelic attachment is the back-to-back positioning of sister kinetochores on opposite sides of centromeric chromatin. This geometric positioning exploits the centromeric chromatin to sterically impede kinetochores from attaching to microtubules other than from the pole that they face, thereby promoting the proper attachment of each kinetochore's microtubules emanating from only one spindle pole (Indjeian and Murray 2007; Loncarek et al. 2007). Depletion of $\mathrm{Rb}$ protein suppresses expression of cohesin and condensin subunits that decrease centromere cohesion. As a consequence, interkinetochore distance increases and horseshoe-shaped kinetochore pairs form. This suggests that the normally stiff centromeric chromatin linking sister kinetochores has become 
flexible and no longer effectively screens kinetochores from attaching to spindle microtubules from inappropriate directions (Manning et al. 2010). Alterations to the kinetochore itself may also increase the rate of formation of merotelic attachments. For example, Hec1 overexpression increases the incidence of merotely and Hecl levels increase in Rb-deficient cells (DiazRodriguez et al. 2008; Ferretti et al. 2010). Presumably, excess Hecl increases the microtubule binding capacity of kinetochores, but it may also suppress the efficiency with which attachment errors are corrected.

\section{Mechanisms of chromosome structure instability}

In addition to alterations in whole chromosome numbers, cancer cells often contain chromosomes with large structural rearrangements, including deletions, duplications, inversions, isochromosomes, ring structures and marker chromosomes, and unbalanced and balanced translocations. Balanced translocations are frequently associated with leukemias and lymphomas, with specific translocations linked with specific cancers (reviewed in Mitelman et al. 2007; Nambiar et al. 2008). These translocations usually represent the single oncogenic change responsible for these cancers as they create driver mutations that initiate and maintain the cancer cell phenotype. Chromosome structure rearrangements such as translocations in solid tumors can be recurrent; however, the vast majority of structural changes in these contexts appear to be non-recurring and random (Castro et al. 2006). Additionally, unlike some blood cancers which often have only one or two recurrent translocations, solid tumors often contain numerous translocations, with different cells in the same tumor containing different structural rearrangements and more complex rearrangements suggestive of multiple rounds of chromosome breakage and fusion. Some of these rearrangements have been found to amplify oncogenes or delete copies to tumor suppressor genes, which supports a causative role in tumorigenesis. However, it remains unknown if the majority of these structural rearrangements have a causative role in solid tumors, or if they are merely the result of decreased DNA damage checkpoints, DNA repair pathways, and/or mitotic segregation errors.

Recurrent rearrangements such as those found in leukemia and lymphoma influence tumorigenesis by either deregulating expression of specific target genes or by producing a hybrid, chimeric gene through fusion of parts of two genes on separate chromosomes (Mitelman et al. 2007). For example, Burkitt's lymphoma is associated with a translocation that places the MYC gene adjacent to one of three immunoglobulin loci, most commonly the immunoglobulin heavy chain locus in the translocation involving chromosomes 8 and 14 [t(8;14)(q23;q32)] (Taub et al. 1982). The consequence of these translocations is the hyperexpression of c-myc by the potent enhancer that ordinarily regulates immunoglobulin gene expression in B cells. Overexpression of translocated genes driven by immunoglobulin enhancers occurs in a large percentage of B-cell malignancies but not other types of cancer, suggesting that these translocations arise through aberrant rearrangement of immunoglobulin genes that are required to produce antibody diversity (Küppers 2005). An example of a chimeric gene formed through chromosomal translocation is BCR-Abl generated by the translocation of chromosomes 9 and $22[\mathrm{t}(9 ; 22)(\mathrm{q} 34$; q11)] found in $95 \%$ of patients with chronic myelogenous leukemia (Nowell and Hungerford 1960; Kurzrock et al. 2003). The chimeric protein created by this translocation retains Abl kinase activity, but that activity is no longer appropriately regulated. Recurrent translocations that generate chimeric fusions play a large role in driving tumorigenesis in blood cancers (Mitelman et al. 2007; Nambiar et al. 2008). Recurrent gene fusions may also play a role in tumorigenesis in solid tumors; however, they appear to be quite rare with perhaps the exception of prostate cancer (Tomlins et al. 2005; Mitelman et al. 2007).

In the last decade, spectral karyotyping (SKY), which labels each chromosome with a unique probe set, has allowed the in-depth characterization of the translocations that occur in solid tumors. SKY karyotyping can identify the presence of a chromosome if $>1 \mathrm{Mb}$ is present. Smaller scale chromosomal rearrangements can be detected using array comparative genomic hybridization (CGH; Bayani and Squire 2001). Evaluating cell lines derived from solid tumors represented in the NCI-60 panel with these tools revealed 15 clonal translocations per cell line, on average (Roschke et al. 2003). This number is comparable to other studies using cancer cell lines as well as numerous studies that have examined karyotypes in a variety of tumor types including 
prostate, breast, lung, colon, and brain (Davidson et al. 2000; Abdel-Rahman et al. 2001; Castro et al. 2006). Close examination of the chromosome rearrangements found in these solid tumors reveals a high level of complexity, often with portions of four or more chromosomes found on the same mosaic chromosome providing evidence of repeated breakage and fusion resulting in regions of amplification or deletion. Most of these rearrangements are not found in more than one cancer or all cells within a single cancer, and are unbalanced (meaning the reciprocal translocation is not present), which suggests these rearrangements form through a random mechanism and that these structural changes are ongoing during tumor cell growth (Castro et al. 2006). We refer to this ongoing genomic instability as chromosome structure instability (CSI). Thus, the karyotypes of solid tumors are far more complex than the single chromosome aberrations that drive many leukemias or lymphomas. The impact of these highly rearranged chromosomes on cancer initiation or promotion remains unknown. In principle, these rearrangements may alter the expression of genes adjacent to the breakpoints or create chimeric fusion proteins, although the number of translocations and rearrangements and the point of breakage/fusion differ from cancer to cancer make it unlikely that these create driver mutations. Since the majority of these translocations are unbalanced, it is more likely that they generate partial or segmental aneuploidy. Interestingly, comparisons of aneuploid and diploid colon cancer cell lines revealed that aneuploid cell lines have greater numbers of chromosome structure rearrangements raising the intriguing possibility that there is a relationship between CIN and CSI (Abdel-Rahman et al. 2001).

Chromosomal translocations arise through inappropriate repair of DNA double strand breaks. Developing tumors often go through a period of elevated rates of chromosome structure change termed the breakage-fusion-bridge cycle (Gisselsson et al. 2000). This occurs when the number of telomere repeats (6-bp-repeat sequences which cap the ends of each chromosome-in humans, telomeres become shorter after each division) drop below a critical threshold where they no longer prevent chromosomes from fusing to each other, leading to the formation of dicentric (possessing two centromeres) chromosomes. Dicentrics form bridges between daughter cells during late mitosis and frequently break during abscission.
DNA double strand break repair mechanisms then fuse the broken chromosomes generating chromosomal translocations that are, again, dicentric, and this cycle continues for several cell cycles (Gisselsson et al. 2000, 2001). Cancer cells most often overcome this cycle by activation of telomerase, but only after several rounds of chromosome breakage leading to CSI as judged by the high numbers of chromosomal translocations (Lo et al. 2002). It is thought that mutations in proteins that permit cell cycle progression in the presence of double stranded breaks (such as mutations in p53, BRCA1, BRCA2, ATM, and ATR) may facilitate CSI (Lengauer et al. 1998).

CSI is most often associated with cancer, although this type of instability also occurs in normal tissues of individuals with rare autosomal recessive disorders including Bloom's syndrome, ataxia telangiectasia, and Fanconi anemia (Duker 2002). Bloom's syndrome is caused by mutations in the BLM gene (which encodes a helicase; Ellis et al. 1995). Loss of function of the BLM protein greatly increases the frequency of DNA double strand breaks leading to sister chromatid exchange as well as chromosome breakage, translocations, and other structural abnormalities (Wang et al. 2000). Ataxia telangiectasia is caused by mutations in ATM which encodes an important protein in detecting and repairing double strand breaks. Loss of ATM allows cells to progress through the cell cycle in the presence of double stranded breaks and greatly enhances the frequency of CSI (Savitsky et al. 1995; Lavin 2008). Fanconi anemia is caused by mutations in one of 13 FANC genes. Loss of FANC proteins disrupts a pathway involved in repair of DNA damage sustained during replication or from reactive oxygen species (Moldovan and D'Andrea 2009). The phenotypes associated with these mechanisms for CSI are differentFanconi anemia patients have a short stature and are often born with birth defects including polydactyly and microcephaly; ataxia telangiectasia patients suffer from poor coordination, thin blood vessels, and weakened immune systems; and Bloom's syndrome is characterized by short stature, skin rashes that appear after exposure to sun, and areas of hypo- and/ or hyperpigmentation-yet they are all associated with elevated cancer susceptibility, with leukemias, lymphomas, and solid tumors occurring with early onset (Duker 2002). Efforts to mimic CSI in mouse models has shown that mice with mutant Blm alleles 
develop a wide variety of tumors (Luo et al. 2000). Additionally, mice with combined telomerase deficiency and knockout or heterozygosity of p53 develop epithelial tumors at an increased rate (Artandi et al. 2000), most likely due to repeated rearrangements in specific regions that result in amplification of oncogenes/deletion of tumor suppressors (O'Hagan et al. 2002; Maser et al. 2007).

\section{Consequences of chromosome aberration}

The adult human has approximately 100 trillion cells with about a hundred million cells dividing at any given time (Alberts et al. 2002; Jorde et al. 2010). It has been shown that diploid human cell lines grown in culture mis-segregate a chromosome about once every hundred cell divisions (Cimini et al. 1999; Thompson and Compton 2008). If the missegregation rate were equivalent in somatic tissues, then it would seem that these tissues would quickly become populated with aneuploid cells, yet this does not occur. In most healthy somatic tissues (exceptions being megakaryocytes, hepatocytes, and some neurons), aneuploid cells do not proliferate efficiently and the overall karyotype of the cell population remains diploid. However, cancer cells are highly aneuploid with numerous copy number alterations (Fig. 1) and do not appear to suffer a limitation in proliferation. This suggests that mechanisms exist to prevent aneuploid cell proliferation and cancer cells must overcome this mechanism to propagate efficiently following chromosome mis-segregation.

One candidate that would suppress aneuploid cell growth is the tumor suppressor p53. Support for this hypothesis comes from the strong positive correlation between mutation of p53 and aneuploid tumors (De Angelis et al. 1993; Meling et al. 1993; Campomenosi et al. 1998; Duensing and Duensing 2005; Schjolberg et al. 2009). In addition, p53 mutations often occur before aneuploidy (Blount et al. 1994), p53 is inactivated at the same time as aneuploidization in the immortalization process (Harvey et al. 1993), and somatic tissues in p53 null mice have elevated levels of aneuploidy (Fukasawa et al. 1997). Direct evidence linking p53 to aneuploidy intolerance comes from experiments where diploid colorectal cancer cells were induced to mis-segregate chromosomes by elevating levels of merotely. Aneuploid cells that formed in these populations arrested in the cell cycle with elevated levels of p53 and the cyclin kinase inhibitor p21 (Thompson and Compton 2010). Elimination of p53 function allowed for aneuploid cell proliferation, suggesting that the p53 pathway prevents aneuploid cell growth and that aneuploidy itself is not detrimental to cell viability. Aneuploid cell survival after disruption of the mitotic checkpoint also induced p53 dependent response to aneuploidy; however, in checkpoint defective cells reactive oxygen species and activation of ATM were responsible for activation of p53 ( $\mathrm{Li}$ et al. 2010). Activation of p53 is commonly associated with DNA damage, but in both studies no DNA damage was detected as judged by staining for gamma-H2AX foci, indicating that $\mathrm{p} 53$ is being activated in the absence of DNA double stranded breaks ( $\mathrm{Li}$ et al. 2010; Thompson and Compton 2010). Inhibition of the p38 stress kinase also allowed aneuploid cell growth leading to a model where chromosome mis-segregation induces a stress response that activates the p53 pathway to block cell cycle progression. Cellular stress could be caused by imbalances in protein expression caused by improper dosage for genes on the aneusomic chromosome (Thompson and Compton 2010). Evidence for protein expression imbalances was recently provided through proteomic analyses of aneuploid strains of budding yeast (Pavelka et al. 2010).

Mutation of p53 is not the only way to gain tolerance for an aneuploid genome as aneuploid neurons and polyploid/aneuploid hepatocytes are likely to be wild type for p53 (Rehen et al. 2001; Duncan et al. 2010). Also, some aneuploid tumors are wild type for $\mathrm{p} 53$, and aneuploid embryonic cells divide for many generations before embryonic lethality (http://www.sanger.ac.uk/perl/genetics/CGP/cosmic; Dobles et al. 2000; Kalitsis et al. 2000). There are multiple ways to inhibit the p53 pathway without directly mutating the p53 gene including MDM2/ MDM4 overexpression, deletion or promoter hypermethylation of $\mathrm{p} 14^{\mathrm{ARF}}$, inhibition of upstream activators such as p38 stress kinase or inhibition of downstream targets (Esteller et al. 2000; Toledo and Wahl 2006; Mikhailov et al. 2007; Ventura et al. 2007; Vousden and $\mathrm{Lu}$ 2007). In the context of cancer cells, it is likely that tolerance for aneuploidy is provided by either direct mutation of $\mathrm{p} 53$ or the disruption of the p53 pathway through these other routes since the p53 pathway is disrupted in most human cancers. In contrast, it is unlikely that aneuploid neurons, 
hepatocytes, and embryonic cells have lost p53 function, suggesting that alternative mechanisms for aneuploidy tolerance exist. Intriguingly, normal hepatocytes are polyploid and frequently aneuploid because they mis-segregate chromosomes at high rates (Duncan et al. 2010). Since aneuploidy and CIN appear to play a role in tumorigenesis, it remains unknown how genomically unstable hepatocytes evade oncogenic transformation.

Whether whole chromosome aneuploidy and partial aneuploidy caused by translocation are causes or consequences of deregulated cell growth and cancer has been a matter of debate for decades. A significant amount of work has gone into the generation of mouse models of aneuploidy/CIN where chromosome missegregation is induced by partial inhibition of the mitotic checkpoint. Many of these mouse models including heterozygous deletions of Mad1, Mad2, and CENP-E, and hypomorph alleles of Bub1 develop tumors late in life, supporting a causative role for aneuploidy in tumorigenesis (recently reviewed in Foijer et al. 2008; Holland and Cleveland 2009). However, some mouse models of aneuploidy/CIN do not develop tumors despite evidence for aneuploidy in tissues. This indicates that tissue context is an important contributing factor to the susceptibility of cells to transformation induced by aneuploidy. Moreover, this underscores the importance of designing experiments in mouse models where the rate of chromosome mis-segregation can be quantified and the roles of aneuploidy and CIN can be determined independently.

The most likely cause of transformation induced by CIN is through loss of heterozygosity for a tumor suppressor or amplification of an activated oncogene. Chromosome loss has been shown to reveal recessive mutations in tumor suppressors (Cavenee et al. 1983), and the late appearance of tumors in mouse models of aneuploidy hints that other mutations must be present for aneuploidy to promote tumorigenesis. Recent mouse models combining mitotic segregation defects with heterozygosity for tumor suppressors support this hypothesis. Reduced Bub1 levels combined with heterozygous deletion of $\mathrm{p} 53$ or the $\mathrm{Apc}^{\mathrm{MIN}}$ allele led to accelerated rates of tumor formation compared to mice heterozygous for the tumor suppressor without chromosome segregation defects (Baker et al. 2009). However, reduced segregation fidelity had no impact on tumors formed in mice heterozygous for $\mathrm{Rb}$, and decreased tumor formation in mice heterozygous for
PTEN (Baker et al. 2009). Likewise, mice heterozygous for CENPE with p19/ARF deleted or treated with DNA damage inducing DMBA had decreased rates of tumor formation (Weaver et al. 2007). These results again point to tissue context as an important component for cellular transformation. An alternative hypothesis is that aneuploidy imbalances the dosage of the gene products on the aneusomic chromosome which can be hundreds of proteins. Dosage changes in several cell cycle regulators may have large consequences on the cells' commitment to cell cycle progression. However, this model seems inconsistent with mouse models that harbor heterozygous mutations in checkpoint genes but show no increase in rates of tumor formation despite equivalent levels of aneuploidy and presumably similar rates of chromosome missegregation as other mouse models for aneuploidy. If imbalances in protein levels were sufficient for cell transformation, then Bubl heterozygous mice should have an equivalent rate of tumor formation to CENP-E heterozygous mice because the two strains contain similar levels of aneuploidy, but they do not (Jeganathan et al. 2007; Weaver et al. 2007). Lastly, it has been proposed that aneuploidy causes a "mutator" phenotype: imbalances in the levels of proteins involved in DNA replication and repair could undermine genome fidelity and an increase the DNA mutation rate (Duesberg et al. 2000; Duesberg and Li 2003). Solid tumor formation requires approximately four to seven mutations in tumor suppressors and oncogenes in order to escape proliferation restraints. Large-scale gene sequencing studies suggest even more pathways must be disrupted, as tumors possess mutations in genes involved in nearly all of 12 critical regulatory signal transduction pathways (Jones et al. 2008). Support for this hypothesis comes from the fact that solid cancers are almost always aneuploid, with the exception being tumors with mutations in genes involved in nucleotide excision repair (NER) or mismatch repair (MMR). Tumors with disrupted NER or MMR pathways have elevated rates of mutation called microsatellite instability (MIN). MIN cancer cells are often near diploid and possess fewer structural rearrangements than their aneuploid counterparts (Lengauer et al. 1997; AbdelRahman et al. 2001). In MIN cells, aneuploidy is not a requirement for tumorigenesis; however, non-MIN solid tumors cells are rarely ever diploid, which suggests a necessity for aneuploidy to generate mutations in cells with intact NER and MMR pathways. 
In addition to the potential to promote tumor formation by generating aneuploidy, CIN is correlated with resistance to chemotherapies and metastasis (Swanton et al. 2009; Warth et al. 2009). Frequent mis-segregation of chromosomes constantly shuffles gene dosage and increases the likelihood that karyotypes favorable for growth in the presence of chemotherapeutic drugs will emerge. Moreover, CIN could generate karyotypes that promote other phenotypes associated with advanced stage disease including invasion and growth in new microenvironments. Recent work using budding yeast as a model system demonstrated that some aneuploid strains have a growth advantage relative to their euploid counterparts under stressful growth conditions including the presence of chemotherapeutic drugs (Rancati et al. 2008; Pavelka et al. 2010). Thus, chromosomal changes induced by CSI (high rate of chromosome structure changes) and CIN (high rate of chromosome mis-segregation) provides the driving mechanism that allows cancer cells to sample the genomic landscape to find an aneuploid karyotype (a state of improper chromosome numbers) that may be transformative or best suited for growth in stressful environments.

\section{Conclusions}

Advances in methodology including SKY chromosome painting and array $\mathrm{CGH}$ are revealing the wide spectrum of karyotypic abnormalities in cancer cells. Most solid tumors are genomically unstable and frequently missegregate whole chromosomes (CIN) and suffer ongoing chromosome structure damage (CSI). The mechanisms responsible for CIN are now clear and the mechanisms causing CSI are emerging. This sets the stage to begin rationally testing how $\mathrm{CIN}$ and CSI contribute to cancer initiation and promotion independently of the contribution of the resultant aneuploidy. Inhibition of CIN or CSI could suppress the ability of cancer cells to sample the genomic landscape, and may rob from the cancer cells the very currency they require to adapt to new surroundings and develop resistance to therapy.

Acknowledgments We would like to thank James Denegre (The Jackson Laboratory, Bar Harbor, Maine) for providing the SKY karyotyping. We apologize to those investigators whose work we could not cite due to length constraints. Work in the authors' lab is supported by the National Institutes of Health (GM51542).

\section{References}

Abdel-Rahman WM, Katsura K, Rens W et al (2001) Spectral karyotyping suggests additional subsets of colorectal cancers characterized by pattern of chromosome rearrangement. Proc Natl Acad Sci 98:2538-2543

Alberts B, Johnson A, Lewis J, Raff M, Roberts K, Walter P (2002) Molecular biology of the cell, 4th edn. Garland Science, New York, p 1548

Artandi SE, Chang S, Lee SL et al (2000) Telomere dysfunction promotes non-reciprocal translocations and epithelial cancers in mice. Nature 406:641-645

Baker DJ, Jin F, Jeganathan KB, van Deursen JM (2009) Whole chromosome instability drives tumorigenesis through tumor suppressor gene loss of heterozygosity. Cancer Cell 16:475-486

Bakhoum SF, Thompson SL, Manning AL, Compton DA (2009a) Genome stability is ensured by temporal control of kinetochore-microtubule dynamics. Nat Cell Biol 11:27-35

Bakhoum SF, Genovese G, Compton DA (2009b) Deviant kinetochore microtubule dynamics underlie chromosomal instability. Curr Biol 19:1937-1942

Bayani J, Squire JA (2001) Advances in the detection of chromosomal aberrations using spectral karyotyping. Clin Genet 59:65-73

Bettencourt-Dias M, Glover DM (2007) Centrosome biogenesis and function: centrosomics brings new understanding. Nat Rev Mol Cell Biol 8:451-463

Blount PL, Galipeau PC, Sanchez CA et al (1994) 17p allelic losses in diploid cells of patients with Barrett's esophagus who develop aneuploidy. Cancer Res 54:2292-2295

Campomenosi P, Assereto P, Bogliolo M et al (1998) p53 mutations and DNA ploidy in colorectal adenocarcinomas. Anal Cell Pathol 17:1-12

Castro MA, Onsten TG, Moreira JC, de Almeida RM (2006) Chromosome aberrations in solid tumors have a stochastic nature. Mutat Res 600:150-164

Cavenee WK, Dryja TP, Phillips RA et al (1983) Expression of recessive alleles by chromosomal mechanisms in retinoblastoma. Nature 305:779-784

Choi CM, Seo KW, Jang SJ et al (2009) Chromosomal instability is a risk factor for poor prognosis of adenocarcinoma of the lung: fluorescence in situ hybridization analysis of paraffin-embedded tissue from Korean patients. Lung Cancer 64:66-70

Cimini D, Tanzarella C, Degrassi F (1999) Differences in malsegregation rates obtained by scoring ana-telophases or binucleate cells. Mutagenesis 14:563-568

Cimini D, Fioravanti D, Salmon ED, Degrassi F (2002) Merotelic kinetochore orientation versus chromosome mono-orientation in the origin of lagging chromosomes in human primary cells. J Cell Sci 115:507-515

Cimini D, Moress B, Canman JC, Salmon E (2003) Merotelic kinetochore orientation occurs frequently during early mitosis in mammalian tissue cells and error correction is achieved by two different mechanisms. J Cell Sci 116:4213-4225

Cimini D, Wan XH, Hirel CB, Salmon ED (2006) Aurora kinase promotes turnover of kinetochore microtubules to 
reduce chromosome segregation errors. Curr Biol 16:1711-1718

Davidson JM, Gorringe KL, Chin SF et al (2000) Molecular cytogenetic analysis of breast cancer cell lines. $\mathrm{Br} \mathrm{J}$ Cancer 83:1309-1317

De Angelis PM, Stokke T, Smedshammer L et al (1993) p53 expression in associated with a high degree of tumor DNA aneuploidy and incidence of p53 gene mutation, and is localized to the aneuploid component in colorectal carcinomas. Int J Oncol 3:305-312

Diaz-Rodriguez E, Sotillo R, Schvartzman J, Benezra R (2008) Hecl overexpression hyperactivates the mitotic checkpoint and induces tumor formation in vivo. Proc Natl Acad Sci 105:16719-16724

Dobles M, Liberal V, Scott ML, Benezra R, Sorger PK (2000) Chromosome missegregation and apoptosis in mice lacking the mitotic checkpoint protein Mad2. Cell 101:635-645

Duensing A, Duensing S (2005) Guilt by association? p53 and the development of aneuploidy in cancer. Biochem Biophys Res Commun 331:694-700

Duesberg P, Li R (2003) Multistep carcinogenesis: a chain reaction of aneuploidizations. Cell Cycle 2:202-210

Duesberg P, Rausch C, Rasnick D, Hehlmann R (1998) Genetic instability of cancer cells is proportional to their degree of aneuploidy. Proc Natl Acad Sci 95:13692-13697

Duesberg P, Rasnick D, Li R, Winters L, Rausch C, Hehlmann R (1999) How aneuploidy may cause cancer and genetic instability. Anticancer Res 19:4887-4906

Duesberg P, Stindl R, Hehlmann R (2000) Explaining the high mutation rates of cancer cells to drug and multidrug resistance by chromosome reassortments that are catalyzed by aneuploidy. Proc Natl Acad Sci 97:14295-14300

Duker NJ (2002) Chromosome breakage syndromes and cancer. Am J Med Genet 115:125-129

Duncan AW, Taylor MH, Hickey RD et al (2010) The ploidy conveyor of mature hepatocytes as a source of genetic variation. Nature 467:707-710

Ellis NA, Groden J, Ye TZ et al (1995) The Bloom's syndrome gene product is homologous to RecQ helicases. Cell 83:655-666

Esteller M, Tortola S, Toyota M et al (2000) Hypermethylationassociated inactivation of $\mathrm{p} 14^{\mathrm{ARF}}$ is independent of p16 ${ }^{\mathrm{INK} 4 \mathrm{a}}$ methylation and p53 mutational status. Cancer Res 60:129-133

Ferretti C, Totta P, Fiore M et al (2010) Expression of the kinetochore protein $\mathrm{Hecl}$ during the cell cycle in normal and cancer cells and its regulation by the $\mathrm{pRb}$ pathway. Cell Cycle 9:4174-4182

Foijer F, Draviam VM, Sorger PK (2008) Studying chromosome instability in the mouse. Biochim Biophys Acta 1786:73-82

Fukasawa K, Weiner F, Vande Woude GF, Mai S (1997) Genomic instability and apoptosis are frequent in p53 deficient young mice. Oncogene 15:1295-1302

Ganem NJ, Storchova Z, Pellman D (2007) Tetraploidy, aneuploidy and cancer. Curr Opin Genet Dev 17:157-162

Ganem NJ, Godinho SA, Pellman D (2009) A mechanism linking extra centrosomes to chromosomal instability. Nature 460:278-282

Gao C, Furge K, Koeman J et al (2007) Chromosomal instability, chromo- some transcriptome, and clonal evo- lution of tumor cell populations. Proc Natl Acad Sci 104:8995-9000

Ghadimi BM, Sackett DL, Difilippantonio MJ et al (2000) Centrosome amplification and instability occurs exclusively in aneuploid, but not in diploid colorectal cancer cell lines, and correlates with numerical chromosomal aberrations. Genes Chromosom Cancer 27:183-190

Gisselsson D, Pettersson L, Höglund M et al (2000) Chromosomal breakage-fusion-bridge events cause genetic intratumor heterogeneity. Proc Natl Acad Sci 97:5357-5362

Gisselsson D, Jonson T, Petersen A et al (2001) Telomere dysfunction triggers extensive DNA fragmentation and evolution of complex chromosome abnormalities in human malignant tumors. Proc Natl Acad Sci 98:1268312688

Guardavaccaro D, Frescas D, Dorrello NV et al (2008) Control of chromosome stability by the b-TrCP-REST-Mad2 axis. Nature 452:365-369

Harvey M, Sands AT, Weiss RS et al (1993) In vitro growth characteristics of embryo fibroblasts isolated from p53deficient mice. Oncogene 8:2457-2467

Hauf S, Cole RW, LaTerra S et al (2003) The small molecule Hesperadin reveals a role for Aurora B in correcting kinetochore-microtubule attachment and in maintaining the spindle assembly checkpoint. J Cell Biol 161:281-294

Heilig CE, Loffler H, Mahlknecht U et al (2010) Chromosomal instability correlates with poor outcome in patients with myelodysplastic syndromes irrespectively of the cytogenetic risk group. J Cell Mol Med 14:895-902

Hernando E, Nahle Z, Juan G et al (2004) Rb inactivation promotes genomic instability by uncoupling cell cycle progression from mitotic control. Nature 430:797-802

Holland AJ, Cleveland DW (2009) Boveri revisited: chromosomal instability, aneuploidy and tumorigenesis. Nat Rev Mol Cell Biol 10:478-487

Indjeian VB, Murray AW (2007) Budding yeast mitotic chromosomes have an intrinsic bias to biorient on the spindle. Curr Biol 17:1837-1846

Jallepalli PV, Waizenegger IC, Bunz F et al (2001) Securin is required for chromosomal stability in human cells. Cell 105:445-457

Jeganathan K, Malureanu L, Baker DJ, Abraham SC, van Deursen JM (2007) Bub1 mediates cell death in response to chromosome missegregation and acts to suppress spontaneous tumorigenesis. J Cell Biol 179:255-267

Jelluma N, Brenkman AB, van den Broek NJ et al (2008) Mps1 phosphorylates Borealin to control Aurora B activity and chromosome alignment. Cell 132:233-246

Jones S, Zhang X, Parsons DW et al (2008) Core signaling pathways in human pancreatic cancers revealed by global genomic analyses. Science 321:1801-1806

Jorde LB, Carey JC, Bamshad MJ (2010) Medical genetics, 4th edn. Mosby Elsevier, Philadelphia, p 350

Kalitsis P, Earle E, Fowler KJ, Choo KH (2000) Bub3 gene disruption in mice reveals essential mitotic spindle checkpoint function during early embryogenesis. Genes Dev 12:2277-2282

Khodjakov A, Cole RW, McEwen BF, Buttle KF, Rieder CL (1997) Chromosome fragments possessing only one kinetochore can congress to the spindle equator. J Cell Biol 136:229-240 
Kline-Smith SL, Khodjakov A, Hergert P, Walczak CE (2004) Depletion of centromeric MCAK leads to chromosome congression and segregation defects due to improper kinetochore attachments. Mol Biol Cell 15:1145-1159

Knowlton AL, Lan W, Stukenberg PT (2006) Aurora B is enriched at merotelic attachment sites, where it regulates MCAK. Curr Biol 16:1705-1710

Kurzrock R, Kantarjian HM, Druker BJ, Talpaz M (2003) Philadelphia chromosome-positive leukemias: from basic mechanisms to molecular therapeutics. Ann Intern Med 138:819-830

Küppers R (2005) Mechanisms of B-cell lymphoma pathogenesis. Nat Rev Cancer 5:251-262

Kuukasjärvi T, Karhu R, Tanner M et al (1997) Genetic heterogeneity and clonal evolution underlying development of asynchronous metastasis in human breast cancer. Cancer Res 57:1597-1604

Kwon M, Godinho SA, Chandhok MS et al (2008) Mechanisms to suppress multipolar divisions in cancer cells with extra centrosomes. Genes Dev 22:2189-2203

Laoukili J, Kooistra MR, Brás A, Kauw J, Kerkhoven RM, Morrison A, Clevers H, Medema RH (2005) FoxM1 is required for execution of the mitotic programme and chromosome stability. Nat Cell Biol 7:126-136

Lavin MF (2008) Ataxia-telangiectasia: from a rare disorder to a paradigm for cell signalling and cancer. Nat Rev Mol Cell Biol 9:759-769

Lengauer C, Kinzler KW, Vogelstein B (1997) Genetic instability in colorectal cancers. Nature 386:623-627

Lengauer C, Kinzler KW, Vogelstein B (1998) Genetic instabilities in human cancers. Nature 396:643-649

Li M, Fang X, Baker DJ et al (2010) The ATM-p53 pathway suppresses aneuploidy-induced tumorigenesis. Proc Natl Acad Sci 107:14188-14193

Lingle WL, Barrett SL, Negron VC et al (2002) Centrosome amplification drives chromosomal instability in breast tumor development. Proc Natl Acad Sci 99:1978-1983

Lo AW, Sabatier L, Fouladi B, Pottier G, Ricoul M, Mumane JP (2002) DNA amplification by breakage/fusion/bridge cycles initiated by spontaneous telomere loss in a human cancer cell line. Neoplasia 4:531-538

Loncarek J, Kisurina-Evgenieva O, Vinogradova T et al (2007) The centromere geometry essential for keeping mitosis error free is controlled by spindle forces. Nature 450:745749

Luo G, Santoro IM, McDaniel LD et al (2000) Cancer predisposition caused by elevated mitotic recombination in Bloom mice. Nat Genet 26:424-429

Maffini S, Maia AR, Manning AL et al (2009) Motorindependent targeting of CLASPs to kinetochores by CENP-E promotes microtubule turnover and poleward flux. Curr Biol 19:1566-1572

Maney T, Hunter AW, Wagenbach M, Wordeman L (1998) Mitotic centromere-associated kinesin is important for anaphase chromosome segregation. J Cell Biol 142:787-801

Manning AL, Longworth MS, Dyson NJ (2010) Loss of pRB causes centromere dysfunction and chromosomal instability. Genes Dev 24:1364-1376

Maser RS, Choudhury B, Campbell PJ et al (2007) Chromosomally unstable mouse tumours have genomic alterations similar to diverse human cancers. Nature 447:966-971
McClelland SE, Burrell RA, Swanton C (2009) Chromosomal instability: a composite phenotype that influences sensitivity to chemotherapy. Cell Cycle 8:3262-3266

Meling GI, Lothe RA, Borresen AL et al (1993) The TP53 tumor suppressor gene in colorectal carcinomas. II. Relation to DNA ploidy pattern and clinicopathological variables. Br J Cancer 67:83-98

Michel LS, Liberal V, Chatterjee A et al (2001) MAD2 haploinsufficiency causes premature anaphase and chromosome instability in mammalian cells. Nature 409:355-359

Mikhailov A, Patel D, McCance DJ, Rieder CL (2007) The G2 p38-mediated stress-activated checkpoint pathway becomes attenuated in transformed cells. Curr Biol $17: 2162-2168$

Mitelman F, Johansson B, Mertens F (2007) The impact of translocations and gene fusions on cancer causation. Nat Rev Cancer 7:233-245

Moldovan GL, D'Andrea AD (2009) How the Fanconi anemia pathway guards the genome. Ann Rev Genet 43:223-249

Nambiar M, Kari V, Raghavan SC (2008) Chromosomal translocations in cancer. Biochim Biophys Acta 1786:139-152N

Nowell PC, Hungerford DA (1960) Chromosome studies on normal and leukemic human leukocytes. J Natl Cancer Inst 25:85-109

O'Hagan RC, Chang S, Maser RS et al (2002) Telomere dysfunction provokes regional amplification and deletion in cancer genomes. Cancer Cell 2:149-155

Parsons DW, Jones S, Zhang X et al (2008) An integrated genomic analysis of human glioblastoma multiforme. Science 321:1807-1812

Pavelka N, Rancati G, Zhu J et al (2010) Aneuploidy confers quantitative proteome changes and phenotypic variation in budding yeast. Nature. doi:10.1038/nature09529

Pereira AL, Pereira AJ, Maia ARR et al (2006) Mammalian CLASP1 and CLASP2 cooperate to ensure mitotic fidelity by regulating spindle and kinetochore function. Mol Biol Cell 17:4526-4542

Quintyne NJ, Reing JE, Hoffelder DR, Gollin SM, Saunders WS (2005) Spindle multipolarity is prevented by centrosomal clustering. Science 307:127-129

Rancati G, Pavelka N, Fleharty B et al (2008) Aneuploidy underlies rapid adaptive evolution of yeast cells deprived of a conserved cytokinesis motor. Cell 135:879-893

Rehen SK, McConnell MJ, Kaushal D, Kingbury MA, Yang AH, Chun J (2001) Chromosomal variation in neurons of the developing and adult mammalian nervous system. Proc Natl Acad Sci 98:13361-13366

Roschke AV, Tonon G, Gehlhaus KS et al (2003) Karyotypic complexity of the NCI-60 drug-screening panel. Cancer Res 15:8634-8647

Salmon ED, Cimini D, Cameron LA, DeLuca JG (2005) Merotelic kinetochores in mammalian tissue cells. Phil Trans R Soc 360:553-568

Savitsky K, Bar-Shira A, Gilad S et al (1995) A single ataxia telangiectasia gene with a product similar to PI-3 kinase. Science 268:1749-1753

Schjolberg A, Clausen OPF, Burum-Auensen E, De Angelis P (2009) Aneuploidy is associated with TP53 expression but not with BRCA1 or TERT expression in sporadic colorectal cancer. Anticancer Res 29:4381-4388 
Silkworth WT, Nardi IK, Scholl LM, Cimini D (2009) Multipolar spindle pole coalescence is a major source of kinetochore mis-attachment and chromosome missegregation in cancer cells. PLoS ONE 4:e6564

Sotillo R, Hernando E, Diaz-Rodriguez E et al (2007) Mad2 overexpression promotes aneuploidy and tumorigenesis in mice. Cancer Cell 11:9-23

Swanton C, Nicke B, Schuett M et al (2009) Chromosomal instability determines taxane response. Proc Natl Acad Sci 106:8671-8676

Taub R, Kirsch I, Morton C et al (1982) Translocation of the cmyc gene into the immunoglobulin heavy chain locus in human Burkitt lymphoma and murine plasmacytoma cells. Proc Natl Acad Sci 79:7837-7841

Thompson SL, Compton DA (2008) Examining the link between chromosomal instability and aneuploidy in human cells. J Cell Biol 180:665-672

Thompson SL, Compton DA (2010) Proliferation of aneuploid human cells is limited by a p53-dependent mechanism. J Cell Biol 188:369-381

Thompson SL, Bakhoum SF, Compton DA (2010) Mechanisms of chromosomal instability. Curr Biol 20:R285-R295

Toledo F, Wahl GM (2006) Regulating the p53 pathway: in vitro hypotheses, in vivo veritas. Nat Rev Cancer 6:909-923

Tomlins SA, Rhodes DR, Perner S et al (2005) Recurrent fusion of TMPRSS2 and ETS transcription factor genes in prostate cancer. Science 310:644-648
Ventura JJ, Tenbaum S, Perdiguero E et al (2007) p38 $\alpha$ MAP kinase is essential in lung stem and progenitor cell proliferation and differentiation. Nat Genet 39:750758

Vousden KH, Lu X (2007) Live or let die: the cell's response to p53. Nat Rev Cancer 2:594-604

Wang W, Seki M, Narita Y et al (2000) Possible association of BLM in decreasing DNA double strand breaks during DNA replication. EMBO J 19:3428-3435

Warth A, Herpel E, Krysa S et al (2009) Chromosomal instability is more frequent in metastasized than in nonmetastasized pulmonary carcinoids but is not a reliable predictor of metastatic potential. Exp Mol Med 41:349353

Weaver BAA, Silk AD, Montagna C, Verdier-Pinard P, Cleveland DW (2007) Aneuploidy acts both oncogenically and as a tumor suppressor. Cancer Cell 11:25-36

Wood LD, Parsons DW, Jones S et al (2007) The genomic landscapes of human breast and colorectal cancers. Science 318:1108-1113

Yao X, Abrieu A, Zheng Y, Sullivan KF, Cleveland DW (2000) CENP-E forms a link between attachment of spindle microtubules to kinetochores and the mitotic checkpoint. Nat Cell Biol 2:484-491

Zhai Y, Kronebusch PJ, Borisy GG (1995) Kinetochore microtubule dynamics and the metaphase-anaphase transition. J Cell Biol 131:721-734 